RESEARCHARTICLE

\title{
Mechanical and Physical Properties of Experimental Antifungal Denture Base Resins
}

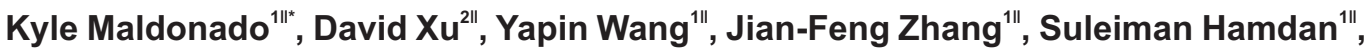 \\ Zezhang T. Wen ${ }^{1}$, Paul L. Fidel Jr ${ }^{3}$, Mairi C. Noverr ${ }^{4}$ and Xiaoming Xu ${ }^{1 *}$
}

'Department of Comprehensive Dentistry and Biomaterials, Louisiana State University - Health Sciences Center, USA

${ }^{2}$ College of Engineering, Louisiana State University, USA

${ }^{3}$ Department of Oral Biology, Louisiana State University - Health Sciences Center, USA

${ }^{4}$ Department of Prosthodontics, School of Dentistry, Louisiana State University - Health Sciences Center, USA

"Authors contributed equally

Open Access

Citation: Maldonado $\mathrm{K}, \mathrm{Xu} \mathrm{D}$, Wang Y, Zhang JF, Hamdan S, Wen ZT, Fidel PL, Noverr MC, Xu X. Mechanical and Physical Properties of Experimental Antifungal Denture Base Resins. Oral Health and Dental Studies. 2018; 1(1):5.

Received: May 14, 2018

Accepted: August 13, 2018

Published: August 29, 2018

Copyright: ๑ 2018 Maldonado K, et al. This is an open access article distributed under the terms of the Creative Commons Attribution License, which permits unrestricted use, distribution, and reproduction in any medium, provided the original author and source are credited.

\section{Corresponding author:}

Kyle Maldonado and Xiaoming Xu, Department of Comprehensive Dentistry and Biomaterials, Louisiana State University - Health Sciences Center, USA.

E-mail: Kylemaldonado90@gmail.com and xxu@lsuhsc.edu

\section{Abstract}

Many denture wearers suffer from fungal infections, particularly Candida albicans, due to failure to properly clean or remove the prosthesis. These infections can cause denture stomatitis, which often leads to irritated or traumatized tissues. Current denture base materials do not have antifungal properties. We have recently synthesized several new antifungal compounds containing 1,4-diazabicyclo [2.2.2] octane (DABCO) (DC16NF, DC11MAF, C2-DC11MAF) that have shown significant antifungal properties. ${ }^{1}$ The purpose of this study is to incorporate these novel antifungal compounds and test mechanical and physical properties of the resulting antifungal materials. The Clotrimazole was also incorporated into denture acrylics and used as a positive control. Samples were then prepared into discs and bars. All groups were tested for flexural strength, flexural modulus, water sorption, and solubility. The results showed that all experimental groups exhibited similar flexural strength as the control, except DC16NF, which had significantly lower strength than the control. DC16NF and DC11MAF both exhibited significantly lower flexural modulus compared to the control while C2DC11MAF exhibited similar flexural modulus as the control. All experimental groups exhibited higher water sorption than the control and a similar or lower solubility as compared to the control. These results suggest that the new antifungal denture base materials possess similar mechanical and physical properties as commercial denture materials, although the formulations can be further optimized.

\section{Keywords}

Denture base, antifungal, flexural strength, water sorption, flexural modulus

\section{Introduction}

Various species of Candida have been identified as harmless commensals of the human oral cavity, but can lead to diseases, such as candidiasis, when the immune system is compromised or the mucosal barriers are broken. Candida species lives primarily on the mucosal surface in biofilms and plays an important role in the development of denture stomatitis by virtue of their ability to adhere to acrylic resin. ${ }^{2}$ Denture stomatitis is considered as the most prevalent and clinically important lesion of denture wearers. The prevalence of denture stomatitis varies, with some report that up to $65 \%$ of denture wearers have this 
condition to some degree,,$^{3,4}$ and about $90 \%$ of cases are associated with Candida species. Early lesions may involve only the palatal vault, although advanced cases cover most of the palate. ${ }^{5}$ The most prevalent site for denture stomatitis is the denture-bearing palatal mucosa. It is unusual for the mandibular mucosa to be involved.

A well-fitting upper denture cuts off the underlying mucosa from the protective action of saliva. In susceptible patients, particularly smokers, this can promote candidiasis, seen as a symptomless area of erythema. The erythema is sharply limited to the area of mucosa occluded by a well-fitting upper denture or even an orthodontic plate. Similar inflammation is not seen under the more mobile lower denture which allows a relatively free flow of saliva beneath it. ${ }^{6}$

Unclean dentures and poor hygiene care are major predisposing factors because healing of the lesions is often seen after meticulous oral and denture hygiene is instituted. However, the tissue surface of the dentures usually shows micropits and microporosities that harbor microorganisms that are difficult to remove mechanically or by chemical cleansing. Since microorganisms penetrate the internal surface to a depth of approximately $1 \mathrm{~mm}$, the acrylic surface of the denture should be removed and relined frequently. A new denture should be provided only when the mucosa has healed and the patient is able to achieve good denture hygiene. ${ }^{2}$ Patients with no symptoms are rarely motivated for treatment, and the infection often persists without the patient being aware of its presence. However, the chronic inflammation may result in increased resorption of the denture-bearing bone. ${ }^{3,4}$

Another approach to treat denture stomatitis is to incorporate anti-fungal drugs into acrylic prosthetic devices in order to prevent the colonization of Candida species. However, this approach does not come without challenges. The anti-fungal drugs incorporated into the prosthetic acrylic must be able to endure the polymerization process and not to negatively affect physical and mechanical properties. The presence of drug may affect the physical properties of the acrylic due to the drug dissolving and resultant porosity. A recent study has shown that incorporating chlorhexidine acetate into heat and cold cured acrylics significantly reduced the hardness and modulus of elasticity. ${ }^{7}$ However, this does not negate the positive effects of incorporating an anti-fungal drug into denture acrylics if the denture acrylics can demonstrate equivalent physical and mechanical properties compared to non-loaded acrylics. On the other hand, as shown by Garcia et al., with the extended presence of the drug at the site of action, less amount of drug is needed to achieve the same therapeutic effect. ${ }^{8}$ The Clotrimazole has been used in denture resins as drug delivery biomaterial to control fungal biofilm, ${ }^{9,10}$ however, there has been no report on their mechanical and physical properties.

We have recently developed several novel antimicrobial compounds containing DABCO (1,4-diazabicyclo[2.2.2]octane). These novel drugs, their structure as shown in Figure 1, are designed for their incorporation into oral prosthetic acrylic to prevent the colonization of Candida albicans and spreading of denture induced stomatitis. ${ }^{9}$ Unlike Clotrimazole which is a releasable antifungal agent, two of the new synthesized DABCO based compounds (DC11MAF, C2-DC11MAF) have polymerizable methacrylate groups capable of forming permanent covalent bonds with acrylic resins bearing the advantages of potential long-term antifungal activities. These positively charged compounds are capable of withstanding the temperature during heat curing of acrylics without comprising antifungal activities. It's imperative to investigate the influence of these compounds on denture acrylics mechanical and physical properties as to determine their possibility for clinical acceptance and commercial applications. Hence, the current study is to test and evaluate the physical and mechanical properties, including flexural strength, flexural modulus, water sorption, and water solubility, of denture acrylics pre-loaded with these novel DABCO compounds. 


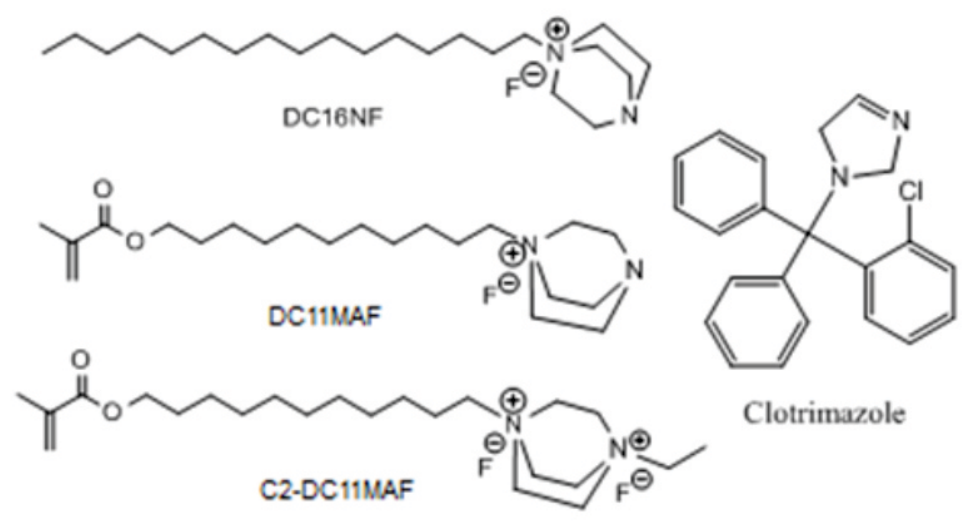

Figure 1. Antifungal compound structure

\section{Materials and Methods}

Three antifungal compounds containing 1,4-diazabicyclo [2.2.2] octane (DABCO) were synthesized. The commercial antifungal drug Clotrimazole was included as positive control. Reacting cetyl bromide $(21.3737 \mathrm{~g}, 70 \mathrm{mmol})$ and octodecyl bromide $(23.3373 \mathrm{~g}, 60 \mathrm{mmol})$ with DABCO produced DC16. Ethyl acetate, the solvent and mixture, were stirred at room temperature for 24 hours, filtered, washed, and dried. DC16F was then synthesized by reacting DC16 with aqueous silver fluoride.

DC11MAF was synthesized using a $250 \mathrm{~mL}$ round bottom flask equipped with magnetic stirring bar which was charged with DABCO (10 g, $40 \mathrm{mmol}), 11$-bromoundecan-1-ol (10 g, 40 $\mathrm{mmol}$ ), and ethyl acetate $120 \mathrm{~mL}$. The reaction mixture was stirred at room temperature overnight. The resulting white solid was precipitated, washed with cold ethyl acetate, and dried in a vacuum oven to give $9.9 \mathrm{~g}, 34 \mathrm{mmol}$, provided a $87.5 \%$ yield. ES-MS analysis (in $\mathrm{MeOH}$, positive ions), $\mathrm{m} / \mathrm{z}=283.0,(\mathrm{M}+\mathrm{H})+$, calculated 283.2749. The intermediate synthesized above $(8.49 \mathrm{~g}, 30 \mathrm{mmol})$ and dichloromethane $(100 \mathrm{~mL})$ were added to a $250 \mathrm{~mL}$ round flask and cooled at $0^{\circ} \mathrm{C}$ for $15 \mathrm{~min}$. Methacryloyl chloride $(31 \mathrm{mmol})$ was added dropwise via a syringe over $10 \mathrm{~min}$. The reaction mixture was stirred at $0^{\circ} \mathrm{C}$ for 2 hours and at room temperature overnight. The reaction was quenched by adding saturated aqueous $\mathrm{K}_{2} \mathrm{CO}_{3}(150$ $\mathrm{mL})$. The aqueous layer was extracted with dichloromethane $(30 \mathrm{~mL} \times 3)$. The combined organic extract was washed sequentially with saturated aqueous $\mathrm{NaHCO}_{3}(60 \mathrm{~mL} \times 2)$ and brine $(100 \mathrm{~mL} \times 2)$, dried over anhydrous $\mathrm{MgSO}_{4}$, filtered, and concentrated under reduced pressure by a rotary evaporator. The crude product was purified on silica gel column with $\mathrm{CH}_{3} \mathrm{COOC}_{2} \mathrm{H}_{5}: \mathrm{CH}_{3} \mathrm{OH}(3: 1)$ as a mobile phase. The purified product $(10.75 \mathrm{~g}, 25 \mathrm{mmol})$ represented a yield of $83 \%$. ES-MS analysis (in $\mathrm{MeOH}$, positive ions): $\mathrm{m} / \mathrm{z}=351.0$, calculated 351.3006. DC11MABr was converted to DC11MAF by reaction with $\mathrm{AgF}(\mathrm{aq})$.

Reacting DC11MABr with ethyliodide using acetonitrile as the solvent produced C2DC11MAF. The product was reflexed 3-5 days until the reaction was complete. TLC was used for confirmation. The purification method used above was also used with this product. ES-MS analysis (in $\mathrm{MeOH}$, positive ions): $\mathrm{m} / \mathrm{z}=379.2$, calculated 380.34 . The C2DC11MABr was further reacted with $\mathrm{AgF}(\mathrm{aq})$ to be converted to C2DC11MAF.

Ivocap High Impact (Ivoclar Vivadent) was used as the control group, or Group 1. The four experimental groups are: Group 2: $5 \%$ Clotrimazole $+5 \%$ di (ethylene glycol) dimethacrylate (DEGDMA); Group 3: DC16NF; Group 4: DC11MAF + 5\% DEGDMA; Group 5: C2DC11MAF + 5\% DEGDMA.

DABCO derivatives were added to the liquid monomer of denture acrylic base material (Ivocap High Impact, Ivoclar Vivadent). The liquid component was then mixed with the powder component. Bar samples and disc samples were then made by injecting the liquid and powder mix into a flask containing a gypsum mold with negative replicas. The bars specimens $(n=12$ each group) were made into $2 \times 2 \times 25 \mathrm{~mm}$ for test of flexural strength and modulus, and discs ( $n=5$ each group) with dimensions approximately $2 \mathrm{~mm}$ in height and $11 \mathrm{~mm}$ in diameter were prepared for water sorption and solubility test (Figure 2). The samples were heat cured $\left(100^{\circ} \mathrm{C}, 2\right.$ hours $)$, polished with 600 grit SiC paper, and measured for dimensions using a digital micrometer with resolution of $0.01 \mathrm{~mm}$. 
Flexural strength and modulus of the bar specimens were tested using three-point flexural test using the Instron 5566 testing machine (Figure 2C) after 24 hours storage in deionized water at $37^{\circ} \mathrm{C}$. Flexural strength is defined as the force per unit area at the instant of fracture in a test specimen subjected to flexural loading..$^{11,12}$ Flexural modulus is computed as the ratio of stress to strain in flexural deformation, or the tendency for a material to bend. ${ }^{13}$

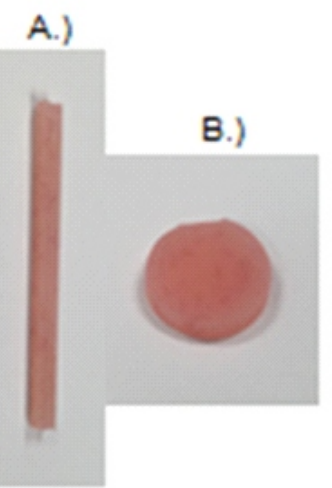

c.)

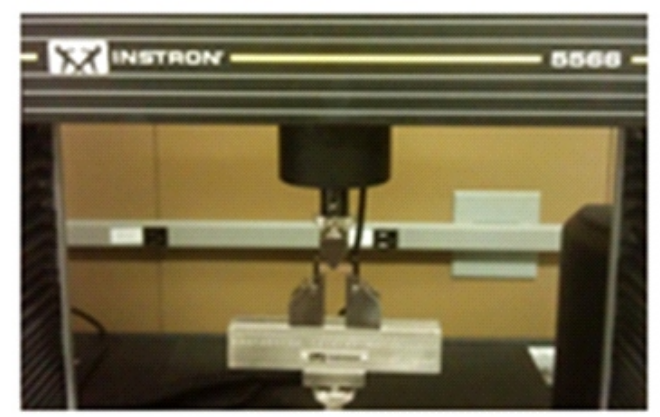

Figure 2. Bar specimens for flexural test (A) and discs samples for water sorption/solubility test (B), and the Intron machine for mechanical test (C)

Water sorption and solubility of the specimens were measured on disc samples. Briefly, disc samples were weighed $\left(\mathrm{m}_{1}\right)$ and immersed in $10 \mathrm{~mL}$ deionized water for 7 days, removed from water, waved in the air for 15 seconds and weighed $\left(m_{2}\right)$. Then, they were placed in a desiccator for 24 hours, weighed every day until constant weight $\left(\mathrm{m}_{3}\right)$. The water sorption Wsp $=\left(m_{2}-m_{1}\right) / A$. Water solubility Wsl $=\left(m_{1}-m_{3}\right) / A$. A is the surface area.

Statistical analysis: The data was analyzed using two-way ANOVA and Tukey post hoc test with significance level set at $95 \%(\alpha=0.05)$.

\section{Results}

\section{Flexural Strength and Modulus}

Flexural strength and modulus of cured denture acrylics containing novel antifungal compounds developed by LSUHSC School of Dentistry were tested along with the positive control containing commercially available anti-fungal Clotrimazole. Ivocap High Impact (Ivoclar Vivadent) was used as the control group.

The results (Figure 3) show that Group 3 which contained DC16NF exhibited a significantly lower flexural strength compared to other groups which had similar flexural strengths to the control. All three other groups exhibited statistically similar results compared to the control group. The results (Figure 4) show that Group 3 (DC16) and Group 4 (DC11MAF) exhibited a significant lower flexural modulus $(P<0.05)$. The results for flexural strength and modulus are summarized in the following table:

Table 1. Flexural strength and modulus of the denture acrylics with various antifungal compounds. The groups with the same label letters have no significant difference $(P>0.05)$

\begin{tabular}{|l|c|c|}
\hline & $\begin{array}{c}\text { Flexural Strength } \\
\text { (MPa) }\end{array}$ & $\begin{array}{c}\text { Flexural Modulus } \\
\text { (MPa) }\end{array}$ \\
\hline Control & $81.06 \pm 17.42^{\mathrm{a}}$ & $2165.69 \pm 255.57^{\mathrm{A}}$ \\
\hline Clotrimazole & $86.54 \pm 12.78^{\mathrm{a}}$ & $2088.78 \pm 265.42^{\mathrm{A}}$ \\
\hline DC16NF & $53.19 \pm 10.15^{\mathrm{C}}$ & $1824.08 \pm 272.70^{\mathrm{B}}$ \\
\hline DC11MAF & $86.39 \pm 28.62^{\mathrm{a}}$ & $1716.63 \pm 414.81^{\mathrm{B}}$ \\
\hline C2DC11MAF & $74.50 \pm 16.19^{\mathrm{b}}$ & $2001.14 \pm 317.87^{\mathrm{A}}$ \\
\hline
\end{tabular}




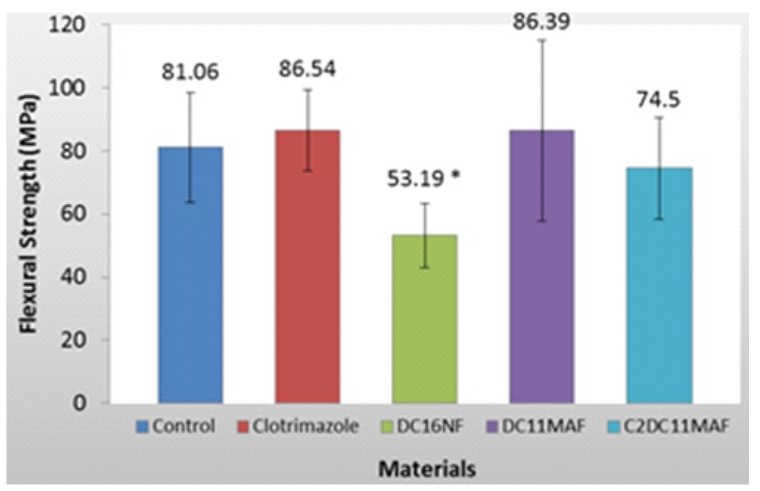

Figure 3. Flexural strength of tested denture base materials. The material with releasable synthesized antifungal compound DC16NF exhibited a significantly lower flexural strength than the other groups $(P<0.05)$

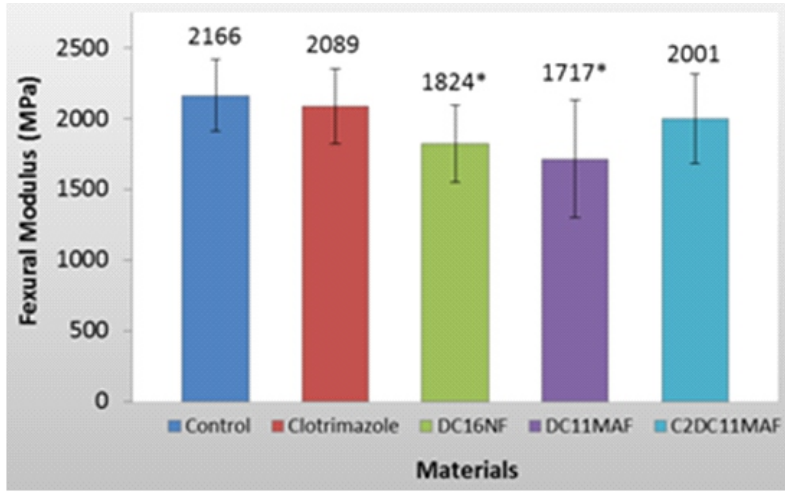

Figure 4. Flexural modulus of tested denture base materials. The materials with releasable synthesized antifungal compound DC16NF and polymerized antifungal compound DC11MAF exhibited significantly lower flexural modulus than the other groups $(P<0.05)$

\section{Water Sorption and Solubility}

All 5 groups were tested for water sorption using the methods described above. The results (Figure 5) show that all experimental groups, including the positive control, exhibited significantly higher water sorption as listed in the following table:

Table 2. Water sorption and solubility of the denture acrylics with various antifungal compounds. The groups with the same label letters have no significant difference $(P>0.05)$

\begin{tabular}{|l|c|c|}
\hline & $\begin{array}{c}\text { Water Sorption } \\
\left(\mathbf{m g} / \mathbf{c m}^{2}\right)\end{array}$ & $\begin{array}{c}\text { Water Solubility } \\
\left(\mathbf{m g} / \mathbf{c m}^{2}\right)\end{array}$ \\
\hline Control & $0.54 \pm 0.04^{\mathrm{a}}$ & $0.85 \pm 0.10^{\mathrm{A}}$ \\
\hline Clotrimazole & $1.55 \pm 0.03^{\mathrm{C}}$ & $0.33 \pm 0.13^{\mathrm{C}}$ \\
\hline DC16NF & $0.95 \pm 0.03^{\mathrm{b}}$ & $0.46 \pm 0.25^{\mathrm{B}, \mathrm{C}}$ \\
\hline DC11MAF & $1.36 \pm 0.18^{\mathrm{C}}$ & $0.58 \pm 0.29^{\mathrm{B}}$ \\
\hline C2DC11MAF & $1.05 \pm 0.08^{\mathrm{b}}$ & $0.48 \pm 0.25^{\mathrm{B}, \mathrm{C}}$ \\
\hline
\end{tabular}

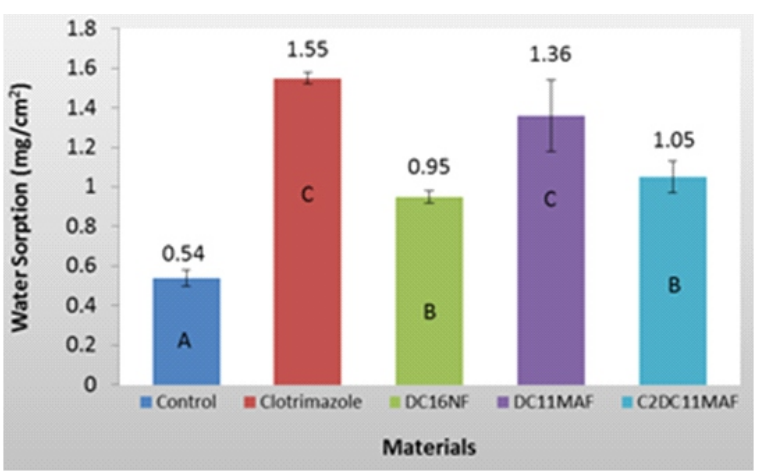

Figure 5. Water sorption of tested denture base materials. All experimental groups exhibited significantly higher water sorption than the control 


\section{Discussion}

Denture stomatitis has a high prevalence among denture wearers. Even in patients with adequate denture cleanliness, fungi, such as Candida sp. can still grow on the prosthesis. We have recently shown that when incorporated in denture base materials, several new antifungal drugs still maintained effective antifungal activities..$^{1,9}$ In this study, we tested the mechanical and physical properties of denture acrylic after incorporating novel anti-fungal drugs into the denture base material and evaluated whether these drugs can be incorporated into the acrylic without compromising the strength of the denture.

In the initial synthesis of DABCO derived compound DC16, bromine was used and caused the compound to be soft and discolored. A likely cause is the oxidation of bromide to bromine during the heat curing process, giving the compound a brownish color. The oxidation also interfered with the polymerization of the acrylic, causing the material to be soft. Fluoride was subsequently used instead of bromine, which produced a pink acrylic. Fluoride was also used to synthesize DC11MAF and C2DC11MAF and produced the same pink color.

\section{Flexural Strength and Modulus}

With regards to flexural strength as seen in Figure 3 and Table 1, Clotrimazole, DC11MAF, and C2DC11MAF all exhibited statistically similar results compared to the control. Clotrimazole and DC11MAF actually had an increased strength compared to the control, while C2DC11MAF exhibited a slightly lower flexural strength. DC16NF exhibited a significantly lower flexural strength than the control groups $(P<0.05)$. However, DC11MAF displayed a large standard deviation which warrants further study.

Clotrimazole and C2DC11MAF also displayed a statistically similar flexural modulus compared to the control. DC16NF and DC11MAF had a significant lower flexural modulus compared to the control as shown in Figure 4 and Table 1. Overall, these results show that DABCO compounds incorporated into denture base resins are capable of providing similar flexural strength and modulus properties compared to commercial denture acrylic currently used. Most importantly, C2DC11MAF and the positive control Clotrimazole both displayed similar flexural strength and flexural modulus compared to the control. This indicates that synthesizing polymerizable denture base resin monomers linked with antimicrobial drugs can be an effective strategy to prevent Candida-associated prosthesis infections.

\section{Water Sorption and Solubility}

Another property tested was water sorption and solubility. Ideally a low water sorption is much desirable because a water saturated acrylic can cause dimensional instability and weaken the mechanical properties of the denture. Water molecules penetrate into the spaces between the hydrophilic polymer chains, pushing them apart and decreasing the secondary bonding forces (van der Waals forces) between the chains, thus acting as plasticizers. This in turn adversely affects the physical and mechanical properties of the resin. ${ }^{14}$ This is an important property to measure because as charged antifungal compounds are added to the denture material, water can be attracted to these charges increasing water sorption. Solubility refers to the resistance to disintegration and dissolution when a material is immersed in water or other solutions, thus, lower water solubility is desirable. ${ }^{15,16}$

In the current study, as we introduce charged antimicrobial compounds into the denture acrylic, water can be attracted to these charged compounds and weaken the dimensional stability of the denture. Further, denture base material should not be excessively water soluble, otherwise water can cause the material to become disintegrated and dissolved when immersed in an aqueous environment. The oral cavity is naturally an aqueous environment therefore water solubility becomes an important property especially since dentures rely on saliva to aid in retention. Our results, however, show that all novel antimicrobial compounds and Clotrimazole exhibited significantly higher water sorption compared to the control. An increased water sorption is expected; however, keeping the water sorption as close to the control as possible is optimal. The ADA standard for water sorption is $0.8 \mathrm{mg} / \mathrm{cm}^{2}$. None of the experimental groups reached that standard. The water solubility results show that all experimental groups were actually significantly lower. However, the ADA standard for water solubility is $0.04 \mathrm{mg} / \mathrm{cm}^{2}$ and the control group had a mean of $0.85 \mathrm{mg} / \mathrm{cm}^{2}$. The significantly increased water solubility in all groups compared to the ADA standard and the large standard deviations also indicate that this test should be repeated. Overall, these results show that 
further optimization of the formulas is required in order to improve the water sorption and water solubility properties.

\section{Conclusion}

The present study demonstrated that the fabricated antifungal denture base materials loaded with DABCO based compounds have similar mechanical and physical properties to commercial denture materials. Combined with the significant antifungal activities on our previous study, these antifungal materials hold potential for clinic denture applications.

\section{Acknowledgement}

This work was supported in part by NIH/NIDCR grants to X. Xu (R01DE019203) and M. Noverr (R01DE022069). 


\section{References}

1. Xu X, Wang Y, Herman JL, et al. Formulation and characterization of novel antimicrobial denture base materials. Frontiers. 2016; 22(11): 1014-1023.

2. Arenas-Arrocena MC, Argueta-Figueroa L, Garcia-Contreras R, et al. New trends for the processing of poly (methyl methacrylate) biomaterial for dental prosthodontics. In: Reddy B, ed. Acrylic Polymers in Healthcare. London: IntechOpen Limited; 2017: 43-74.

3. Reichart PA. Oral mucosal lesions in a representative cross-sectional study of aging Germans. Community Dent Oral Epidemiol. 2000; 28(5): 390-398.

4. Shulman JD, Beach MM, Rivera-Hidalgo F. The prevalence of oral mucosal lesions in U.S. adults: data from the Third National Health and Nutrition Examination Survey 1988-1994. J Am Dent Assoc. 2004; 135(9): 1279-1286.

5. Canger EM, Peruze C, Saadettin K. Denture-related hyperplasia: a clinical study of a Turkish population group. Braz Dent J. 2009; 20(3): 243-248.

6. Cook RJ. Response of the oral mucosa to denture wearing. J Dent. 1991; 19(3):135-147.

7. Addy M., Handley R. The effects of the incorporation of chlorhexidine acetate on some physical properties of polymerized and plasticized acrylics. J Oral Rehabil. 1981; 8(2): 155-163.

8. Garcia CR, Siqueiros A, Benet LZ. Oral controlled release preparations. Pharm Acta Helv.1978; 53(3-4): 99-109.

9. Herman J, Wang Y, Lilly E, et al. Synthesis, antifungal activity, and biocompatibility of novel DABCO compounds and DABCO containing denture base resins. Antimicrob Agents Chemother. 2017; 61: e02575-16.

10. Wen J, Yeh C, Sun Y. Functionalized denture resins as drug delivery biomaterials to control fungal biofilms. ACS Biomater Sci Eng. 2016; 2(2): 224-230.

11. Zweben C, Smith WS, Wardle MW. Test methods for fiber tensile strength, composite flexural modulus, and properties of fabric-reinforced laminates. Composite Materials: Testing and Design (Fifth Conference), 1979.

12. Hashem M, Alsaleem SO, Assery MK, Sukumaran A. A comparative study of the mechanical properties of the anil light-cure and conventional denture base resins. Oral Health Dent Manag. 2014; 13(2):311-315.

13. Campo EA. Chapter 2. Mechanical properties of polymeric materials. In: Selection of Polymeric Materials. William Andrew Inc. 2008; 41-101.

14. Nisar S, Moeen F, Khan YH. Effect of curing regimes on physical properties of heat cure acrylic resin. Pakistan Oral Dental J.2016; 36(1): 156-160.

15. Abdul Mudalip SK, Abu Bakar MR, Jamal P, Adam F, Alam ZM. Molecular recognition and solubility of mefenamic acid in water. Asian J Chem.2016; 28(4): 853-858.

16. Dhillon B,Goyal NK, Malviya R, Sharma PK. Poorly water soluble drugs: Change in solubility for improved dissolution characteristics a review. Global J Pharmacol. 2014; 8(1): 26-35. 